\title{
INFLUENCIA MUTUA DE LA DEFORMACIÓN Y COMPOSICIÓN QUÍMICA SOBRE LA PRECIPITACIÓN INDUCIDA EN ACEROS MICROALEADOS
}

\author{
Alberto Quispe Cohaila'; Sebastian Medina ${ }^{2}$
}

\begin{abstract}
RESUMEN
Mediante ensayos de torsión y usando del método "back extrapolation" se ha determinado la cinética de recristalización estática de aceros microaleados con $V, N b$ y Ti y, a partir de las mismas ha sido posible dibujar los diagramas recristalización-precipitación-tiempo-temperatura (RPTT) además, muestran gráficamente la interacción Recristalización-Precipitación. Estos diagramas muestran que el efecto de la deformación en la cinética de precipitación depende del contenido de microaleante. En este sentido, una nueva expresión se ha propuesto para relacionar la dependencia de la deformación y la composición química con el periodo mínimo de incubación de la precipitación inducida.
\end{abstract}

Palabras Clave: Deformación, microaleados, diagrama RPTT, cinética de precipitación, periodo de incubación.

Influence Mutual of the Deformation and Chemical Composition on Induced Precipitation in Microalloyed Steels

\section{$A B S T R A C T$}

By means of torsion test and applying the "back extrapolation" method, static recrystallization kinetics in microalloyed steels with $\mathrm{V}, \mathrm{Nb}$ and Ti has been determined and, recrystallization-precipitationtime-temperature (RPTT) diagram have been plotted, also, graphically show the RecrystallizationPrecipitation interaction. These diagrams show that the effect of the deformation in the precipitation kinetics depends of the microayolled content. In this sense, new expression are proposed to relate the dependence of the deformation and the chemical composition with the minimum incubation time in the precipitation kinetics.

Keywords: Deformation, microalloyed, RPTT diagram, precipitation kinetic, incubation time.

\section{INTRODUCCIÓN}

La recristalización estática de los aceros microaleados es diferente antes y después de la precipitación inducida por la deformación. En el primer caso todos los elementos están en solución y la cinética de la recristalización ocurre de la misma forma que en los aceros de baja aleación, donde los diferentes elementos de aleación contribuyen en mayor o menor medida a retrasar la recristalización ${ }^{[1-3]}$. A medida que la temperatura desciende se alcanza una temperatura crítica, a partir de la cual la recristalización estática es momentáneamente inhibida por efecto de los precipitados inducidos por la deformación. Esta inhibición momentánea aparece como un "plateau" en las curvas de fracción recristalizada frente al tiempo $\mathrm{o}^{[4]}$. La buena definición de la meseta permite trazar la curva de recristalización-precipitación-tiempo-temperatura (RPTT) y consecuentemente obtener un mayor conocimiento que las condiciones de laminación, ejercen sobre la microestructura de estos aceros ${ }^{[5,6]}$. Antes y después del "plateau", la curva de fracción recristalizada obedece a la ley de Avrami. El límite entre las dos fases se denomina temperatura critica de recristalización estática (SRCT). Hasta muy reciente se consideraba que la influencia de la deformación en la cinética de precipitación era independiente de otras variables, como son el contenido y naturaleza de los microaleantes, temperatura, etc. [7]. Sin embargo, Medina y Col. $\left[{ }^{8-10}\right]$, en trabajos llevados a cabo en aceros microaleados con $\mathrm{Ti}, \mathrm{V}$ y $\mathrm{Nb}$ demostraron experimentalmente que existe una interdependencia de la deformación con el contenido de microaleante sobre el periodo de incubación de la precipitación inducida, adoptando una nueva expresión que difiere conceptualmente de la expresión teórica de Dutta y 
Sellars [7]

\section{MATERIALES $Y$ PROCEDIMIENTO EXPERIMENTAL}

Los aceros fueron fabricados por el proceso de refusión bajo electroescoria (ESR) en una unidad capaz de producir lingotes de $30 \mathrm{Kg}$. En Tabla I se muestra la composición química de los aceros estudiados. Las probetas usadas para torsión, tenian $50 \mathrm{~mm}$ de longitud útil y $3 \mathrm{~mm}$ de radio, donde previamente a la deformación las probetas eran austenizadas a temperaturas muy altas $\left({ }^{3} 1200^{\circ} \mathrm{C}\right)$ durante $10 \mathrm{~min}$. A continuación la temperatura era reducida rápidamente hasta la temperatura de ensayo. Previamente se determinó el tamaño de grano austenítico (Tabla I) a las temperaturas de austenización mediante la técnica de intersección lineal, analizando veinte campos para cada probeta. La técnica dilatométrica fue usada para determinar la temperatura de transformación $\mathrm{A}_{13}$ (Tabla i). Conociendo estas temperaturas de transformación se eligieron temperaturas de ensayo que corresponden a la fase austenita.

La fracción recristalizada fue determinada utilizando el método conocido como "Back Extrapolation" para diferentes temperaturas, deformaciones equivalentes de 0,20 y 0,35 y una velocidad de deformación constante de $3,63 \mathrm{~s}^{-1}(=1000 \mathrm{rev} / \mathrm{min})$. Las deformaciones aplicadas fueron programadas y luego calculadas sobre la superficie cilindrica de la probeta de acuerdo al criterio de Von Misses ${ }^{[11]}$.

\section{RESULTADOS Y DISCUSIÓN}

\subsection{Recristalización Estática}

En los aceros estudiados las curvas de fracción recristalizada $\left(X_{a}\right)$ frente al tiempo algunas curvas muestran la formación de un "plateau", por ejemplo, en la Fig. 1a (acero V5) se observa en las curvas correspondientes a $900^{\circ} \mathrm{C}, 850^{\circ} \mathrm{C}$ y $800^{\circ} \mathrm{C}$ para una deformación de 0,35 . Las curvas correspondientes a temperaturas mayores tienen la forma "sigmoidal" de la ley de Avrami. La precipitación no inhibe la recristalización permanentemente y luego progresa otra vez hasta que se completa, siguiendo la forma "sigmoidal" registrada antes de la formación del "plateau". Por tanto, antes y después del "plateau", la cinética de recristalización obedece a la ley de Avrami.

Sin embargo, los aceros N4 y N7 son diferentes debido a que muestran doble "plateau" como se muestra en la Fig. 1b (acero N7) en las curvas correspondientes a $1050^{\circ} \mathrm{C}$ y $1000^{\circ} \mathrm{C}$ para una deformación de 0,35 . Este fenómeno, se interpreta como la formación de dos tipos de precipitados que comienzan a formarse prácticamente a la misma temperatura. Una vez que la cinética de precipitación de la primera ha finalizado (primer "plateau"), la recristalización continúa, hasta que la segunda precipitación empieza a ocurrir y una vez que finaliza, la recristalización continua hasta el 100\%.

Tabla 1. Composición química de los aceros (\% peso); austenización ( ${ }^{\circ} \mathrm{C} \times 10 \mathrm{~min}$ ); tamaño de grano (D); y temperatura critica de enfriamiento $\left(\mathrm{Ar}_{3}\right.$, a $0,20^{*}$ y $\left.0,25^{\circ} \mathrm{C} / \mathrm{s}\right)$.

\begin{tabular}{|c|c|c|c|c|c|c|c|c|}
\hline Acero & 8 & 81 & in & is & $x+2$ & 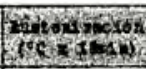 & 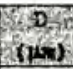 & $\left(C_{3}\right)$ \\
\hline V1 & 0,11 & 0,24 & 1,10 & 0,0105 & $V=0,043$ & 1230 & 172 & $786^{\circ}$ \\
\hline V2 & 0,12 & 0,24 & 1,10 & 0,0123 & $\mathrm{~V}=0,060$ & 1230 & 167 & $782^{*}$ \\
\hline V3 & 0,11 & 0,24 & 1,00 & 0,0144 & $\mathrm{~V}=0,093$ & 1230 & 165 & $784^{*}$ \\
\hline V5 & 0,33 & 0,22 & 1,24 & 0,0146 & $V=0,076$ & 1200 & 165 & 716 \\
\hline V6 & 0,35 & 0,21 & 1,23 & 0,0121 & $V=0,033$ & 1200 & 170 & 715 \\
\hline V8 & 0,38 & 0,22 & 1,42 & 0,0190 & $\mathrm{~V}=0,120$ & 1200 & 157 & 721 \\
\hline $\mathrm{T} 1$ & 0,15 & 0,24 & 1,12 & 0,0105 & $\mathrm{~T}_{\mathrm{i}}=0,021$ & 1230 & 29 & $791^{*}$ \\
\hline $\mathrm{T} 2$ & 0,15 & 0,27 & 1,25 & 0,0100 & $\mathrm{Ti}=0,055$ & 1230 & 95 & $774^{x}$ \\
\hline T3 & 0,15 & 0,26 & 1,10 & 0,0102 & $\mathrm{Ti}=0,075$ & 1230 & 90 & $779 *$ \\
\hline N1 & 0,11 & 0,24 & 1,08 & 0,0058 & $\mathrm{Nb}=0,024$ & 1250 & 210 & $768^{*}$ \\
\hline $\mathrm{N} 2$ & 0,21 & 0,24 & 1,23 & 0,0112 & $\mathrm{Nb}=0,041$ & 1250 & 122 & $786^{x}$ \\
\hline N3 & 0,21 & 0,18 & 1,32 & 0,0119 & $\mathrm{Nb}=0,093$ & 1250 & 116 & 786 \\
\hline N4 & 0,21 & 0,19 & 1,14 & 0,0061 & $\mathrm{Nb}=0,058$ & 1250 & 190 & 679 \\
\hline Nा & 0.29 & 0,22 & 1,30 & 0,0062 & $\mathrm{Nb}=0,058$ & 1295 & 415 & 751 \\
\hline N8 & 0,20 & 0,20 & 1,00 & 0,0056 & $\mathrm{Nb}=0,007$ & 1250 & 140 & 770 \\
\hline N9 & 0,46 & 0,24 & 1,25 & 0,0100 & $\mathrm{Nb}=0,009$ & 1250 & 190 & 704 \\
\hline
\end{tabular}

Fig. 1. Fracción recristalizada $\left(X_{a}\right)$ frente al tiempo (t). Acero (a) V5 v (b) N7
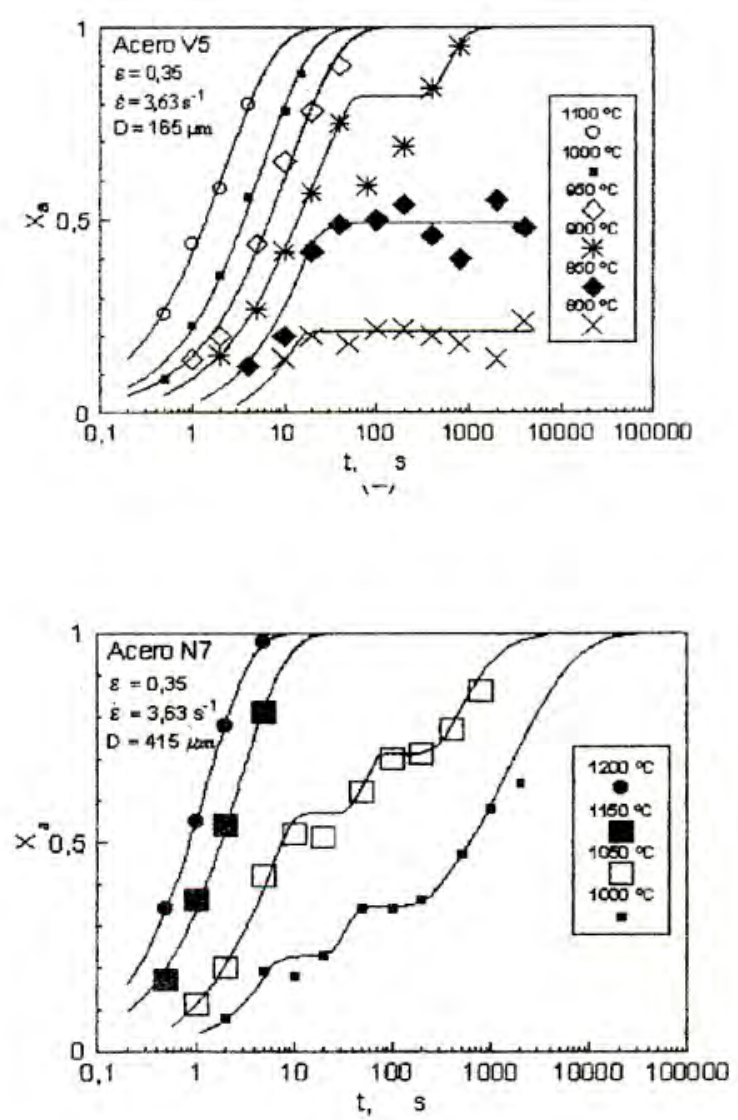


\subsection{Diagramas RPTT}

De las curvas de fracciones recristalizadas $\left(X_{a}\right)$ se determinaron los diagramas RPTT mediante lectura de las temperaturas y tiempos correspondientes a los puntos de intersección de diferentes fracciones recristalizadas $(0,1 ; 0,3 ; 0,5 ; 0,7 ; 0,9$ y 0,95$)$ y, las curvas $\left(P_{5}\right)$ y $\left(P_{f}\right)$ de inicio y final de precipitación, respectivamente, dadas por los puntos de inicio y final de los "plateaus". De este modo se traza los diagramas RPTT como se muestran en la Fig. 2a-b (Aceros V8 y N4). Se observa que la fracción recristalizada no varla entre las curvas de inicio $\left(P_{s}\right)$ y final $\left(P_{f}\right)$ de la precipitación y que esta es representada por una linea horizontal. Una vez que las líneas de cada fracción recristalizada han alcanzado la curva $P_{f}$ continúan descendiendo con menor pendiente. Los diagramas RPTT proporcionan una valiosa información sobre el inicio de la nucleación (nariz de la curva $\mathrm{P}_{5}$ ), es decir cuando el tiempo de incubación de los precipitados $\left(t_{N}\right)$ es mínimo, la fracción de volumen recristalizado es aproximadamente el $50 \%$. Cuando la fracción de volumen recristalizado es menor al $20 \%$, la nucleación de los precipitados es prácticamente imposible.

Fig. 2. Diagrama RPTT. Aceros: (a) V8 y (b) N4

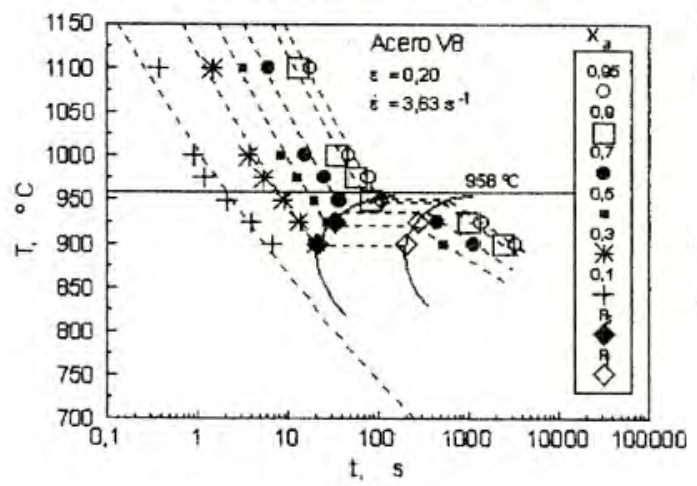

(a)

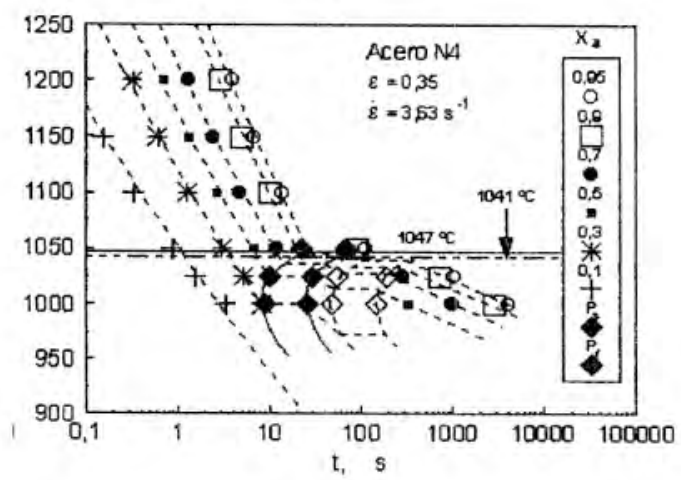

(b)

\subsection{Influencia de la deformación y los elementos aleantes}

De los diagramas RPTT, se deducen el tiempo mínimo de incubación $\left(\mathrm{t}_{\mathrm{N}}\right)$ y el tiempo mínimo del final de la precipitación $\left(t_{N}\right)$ cuyos valores se muestran en Tabla Il: Se observa que los tiempos $t_{\mathrm{N}}$ y $\mathrm{t}_{\mathrm{N}}$ disminuyen con el aumento del contenido de microaleante y deformación. Sin embargo, puede apreciarse que las dos magnitudes no actúan independientemente y el efecto de la deformación en la cinética de precipitación depende del contenido de microaleante. La forma de las curvas RPTT muestran que el modelo establecido por Dutta y Col. $\left[{ }^{8,12}\right]$ es generalmente correcto cualitativamente. Dichos autores establecen que el tiempo $t_{0,05}$ correspondiente para una precipitación parcial de $5 \%$ está relacionada con el contenido de microaleante $(w)$ y deformación (e) cuya relación es:

$$
t_{0,05} \propto w^{-v} \varepsilon^{-\beta}
$$

De acuerdo con la ecuación (2) y aceptando que $t_{0,05}$ y $t_{N}$ son aproximadamente similares, los valores de $b$ fueron determinados y los resultados se recogen en la Tabla II. Se observa que b disminuye con el incremento en el contenido de microaleante en cada grupo de aceros. Realizando un análisis más detallado de la representación gráfica de $b$ frente al contenido (\%peso) del microaleante muestra claramente (Fig. 3) la dependencia de $b$. Es decir, que la disminución del parámetro b con el contenido de microaleante, es debido probablemente a que la influencia de la deformación disminuye gradualmente a medida que las fuerzas de dirección química se incrementan[ $\left.{ }^{\ominus}\right]$. La forma de la curva de regresión sugiere una expresión de tipo Avrami para $b$, debido a que es la más aceptable y es posible la interpretación física. La expresión resultante es la siguiente:

$$
\beta=1,96\left[1-\exp \left(-3,994 \times 10^{-2}\left(\frac{1}{w}\right)^{0,813}\right)\right]
$$

Donde w es el contenido en $\%$ en peso. En Fig. 3 se muestra $b$ frente al contenido de microaleante experimental y calculado de acuerdo a la expresión (2). 
Tabla II. Tiempo minimo ae incubacion $\mathrm{L}_{\mathrm{N}}$, $\mathrm{D}$ experımental $\mathrm{y}$ caicuia...

\begin{tabular}{|c|c|c|c|c|c|c|c|}
\hline Acero & $E$ & $t_{N B},(s)$ & $x_{N m}(x)$ & $42,(3)$ & $\left.t_{\sin }()^{2}\right)$ & $B \infty$ & Atal \\
\hline \multirow{2}{*}{ V1 } & 0,20 & 52 & 340 & - & - & \multirow{2}{*}{0,983} & \multirow{2}{*}{0,790} \\
\hline & 0,35 & 30 & 200 & - & - & & \\
\hline \multirow{2}{*}{ V2 } & 0,20 & 37 & 330 & - & - & \multirow{2}{*}{0,699} & \multirow{2}{*}{0,637} \\
\hline & 0,35 & 24 & 155 & - & - & & \\
\hline \multirow{2}{*}{ v3 } & 0,20 & 20 & 120 & - & - & \multirow{2}{*}{0,400} & \multirow{2}{*}{0,472} \\
\hline & 0,35 & 16 & 115 & - & - & & \\
\hline \multirow{2}{*}{ v5 } & 0,20 & 21 & 180 & - & - & \multirow{2}{*}{0,543} & \multirow{2}{*}{0,544} \\
\hline & 0,35 & 16 & 110 & - & - & & \\
\hline \multirow{2}{*}{ V6 } & 0,20 & 40 & 80 & - & - & \multirow{2}{*}{1,006} & \multirow{2}{*}{0,978} \\
\hline & 0,35 & 23 & 41 & - & - & & \\
\hline \multirow{2}{*}{ V8 } & 0,20 & 19 & 180 & - & - & \multirow{2}{*}{0,422} & \multirow{2}{*}{0,393} \\
\hline & 0,35 & 15 & 90 & - & - & & \\
\hline \multirow{2}{*}{ T1 } & 0,20 & 300 & 1800 & - & - & \multirow{2}{*}{1,960} & \multirow{2}{*}{1,904} \\
\hline & 0,35 & 100 & 600 & - & - & & \\
\hline \multirow{2}{*}{ T2 } & 0,20 & 130 & 300 & - & - & \multirow{2}{*}{1,710} & \multirow{2}{*}{1.104} \\
\hline & 0,35 & 50 & 220 & - & -. & & \\
\hline \multirow{2}{*}{ T3 } & 0,20 & 60 & 150 & -- & $-\cdot$ & \multirow{2}{*}{0,330} & \multirow{2}{*}{0,801} \\
\hline & 0,35 & 50 & 150 & - & - & & \\
\hline \multirow{2}{*}{ N1 } & 0,20 & 24 & 295 & - & - & \multirow{2}{*}{0,770} & 0813 \\
\hline & 0,35 & 16 & 190 & - & - & & 0,013 \\
\hline N2 & 0,20 & 17 & 275 & - & - & 0,580 & 0.472 \\
\hline & 0,35 & 12 & 130 & -- & - & & \\
\hline N3 & 0,20 & 26 & 460 & -- & -- & 0.983 & 1011 \\
\hline & 0,35 & 15. & 200 & - & - & (2, & 1,011 \\
\hline N4 & 0,20 & 11 & 38 & 80 & 230 & 0.592 & 0.652 \\
\hline & 0.35 & 8 & 23 & 42 & 130 & 0,292 & 0,052 \\
\hline 27 & 0.20 & 10 & 35 & 75 & 215 & 0.637 & 0.598 \\
\hline [4) & 0,35 & 7 & 20 & 42 & 110 & 0,031 & 0,270 \\
\hline N8 & 0,20 & 58 & 510 & - & - & 653 & 1,755 \\
\hline $1 \times 0$ & 0,35 & 23 & 220 & - & - & 1,035 & תנ, \\
\hline N9 & 0.20 & 29 & 82 & - & - & 1,502 & 1,649 \\
\hline & 0.35 & 12 & 38 & - & - & & \\
\hline
\end{tabular}

La ecuación (2) sugiere que la deformación inicial influve en la cinética de precipitación cuando el contenido de microaleante es menor a una cierta cantidad, $v$ aue en términos prácticos debe ser aproximadamente $0,3 \%$ en peso. Para contenidos inferiores, el valor de $\mathrm{b}$ debe ser mayor que cero $\mathrm{v}$ consecuentemente la deformación afectará a la cinética de precipitación. Uno de los efectos de la deformación es el incremento de la densidad de dislocaciones, que es origen de la nucleación de los precipitados y este efecto es debilitado gradualmente a medida que las fuerzas de dirección química se incrementan como consecuencia del incremento del contenido de elemento microaleante.

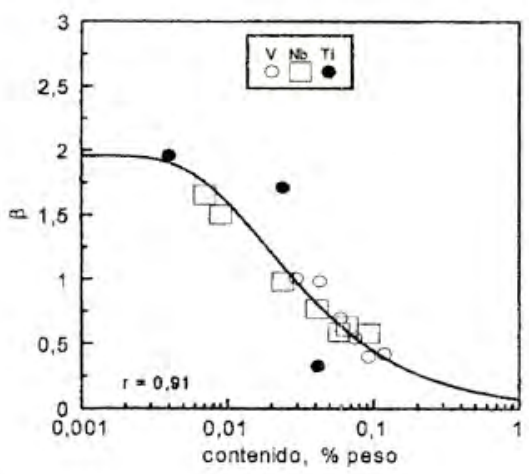

Fig. 3. Parámetro b frente al contenido de $\mathrm{V}, \mathrm{Nb}$ y $\mathrm{Ti}$ (\% peso) para los aceros usados.

\section{CONCLUSIONES}

La cinética de precipitación inducida por la deformación influye en el periodo de nucleación y depende del contenido de elemento microaleante.

La influencia de la deformación en el periodo de incubación para la precipitación disminuye cuando el contenido de microaleante se incrementa.

Para propósitos prácticos se debe considerar que para contenidos mayores a $0,3 \%$ en peso la deformación no tiene influencia en la cinética de precipitación.

- Cuando el contenido de elemento microaleante se aproxima a cero el parámetro $b$ se incrementa, pero no mayor que dos.

\section{AGRADECIMIENTO}

Los autores desean agradecer a la Secretaria de Estado de Educación y Universidades por financiar la estancia en el CENIM del Dr. A. Quispe.

\section{REFERENCIA BIBLIOGRÁFICA}

1. DUTTA, B. and C. M. SELLARS, Mater. Sci. and Technology, 3 3 (1987): 197-207.

2. DUTTA, B.; PALMIERE, E. J. y SELLARS, C. M. Acta Metall. Mater., 49 (2001): 785-794.

3. Faessel, A. Rev. Metall. CIT, 4, (1976): 875-892.

4. LAGNEBORG R.; SIWECKI, T; ZAJAC, S. and HUTCHINSON,B Inst. For Metallforskning, Scand. J. Metall. 28 5, (1999): 186-241

5. KWON, O. ISIJ Int., 32, (1992): 350-358.

6. MEDINA, S.F. and MANCILLA, J. Acta Metall. Mater., 42,12 (1994): 3945-3951

7. Medina, S.F.; Quispe, A.; ISIJ Int., 36, 10 (1996): 1295-1300.

8. MEDINA, S.F; Mater, J. Sci., 32 (1997): 1487-1492

9. Medina, S.F.; Quispe,A.; ISIJ Int., 41, (2001): 774-781.

10. MEDINA, S.F.; QUISPE, A. and Gómez, A.; Mater. Sci. and Technology, 17, 5 (2001): 536-557.

11. QUISPE, A.; MEDINA, S.F. and VALLES, P.; ISIJ Int., 37, (1997): 783-788

12. ZUROB, H.S.; HUTCHINSON, C.R.; BRECHET,Y.; PUDRY,G; Acta Metall. Mater., 50 (2002): 3075-3092. 